\title{
Testing for differences in distribution tails to test for differences in 'maximum' lifespan
}

\author{
Guimin Gao', Wen Wan ${ }^{4}$, Sijian Zhang ${ }^{5}$, David T Redden ${ }^{1,2,3}$ and \\ David B Allison*1,2,3
}

\begin{abstract}
Address: ${ }^{1}$ Department of Biostatistics, Section on Statistical Genetics, University of Alabama at Birmingham, Birmingham, Alabama, USA, ${ }^{2}$ Department of Nutrition Sciences, University of Alabama at Birmingham, Birmingham, Alabama, USA, ${ }^{3}$ Clinical Nutrition Research Center, University of Alabama at Birmingham, Birmingham, Alabama, USA, ${ }^{4}$ Biostatistics and Bioinformatics Unit, Comprehensive Cancer Center, University of Alabama at Birmingham, Birmingham, Alabama, USA and ${ }^{5}$ Department of Biostatistics, University of Alabama at Birmingham, Birmingham, Alabama, USA
\end{abstract}

Email: Guimin Gao - guiming@uab.edu; Wen Wan - wen.wan@ccc.uab.edu; Sijian Zhang - rzhang@uab.edu; David T Redden - samndave@uab.edu; David B Allison* - Dallison@UAB.edu

* Corresponding author

Published: 25 July 2008

BMC Medical Research Methodology 2008, 8:49 doi:10.1186/147I-2288-8-49

This article is available from: http://www.biomedcentral.com/I47I-2288/8/49

(C) 2008 Gao et al; licensee BioMed Central Ltd.

This is an Open Access article distributed under the terms of the Creative Commons Attribution License (http://creativecommons.org/licenses/by/2.0), which permits unrestricted use, distribution, and reproduction in any medium, provided the original work is properly cited.

\begin{abstract}
Background: Investigators are actively testing interventions intended to increase lifespan and wish to test whether the interventions increase maximum lifespan. Based on the fact that one cannot be assured of observing population maximum lifespans in finite samples, in previous work, we constructed and validated several tests of difference in the upper parts of lifespan distributions between a treatment group and a control group by testing whether the probabilities that observations are above some threshold defining 'old' or being in the tail of the survival distribution are equal in the two groups. However, a limitation of these tests is that they do not consider how much above the threshold any particular observation is.
\end{abstract}

Methods: In this article we propose new methods which improve upon our previous tests by considering not only whether an observation is above some threshold, but also the magnitudes by which observations exceed the threshold.

Results: Simulations show that the new methods control type I error rates quite well and that the power of the new methods is usually higher than that of the tests we previously proposed. In illustrative analyses of two real datasets involving rodents, when setting the threshold equal to II 0 (100) weeks for the first (second) datasets, the new methods detected differences in 'maximum lifespan' between groups at nominal alpha levels of 0.01 (0.05) for the first (second) datasets and provided more significant results than competitor tests.

Conclusion: The new methods not only have good performance in controlling the type I error rates but also improve the power compared with the tests we previously proposed.

\section{Background}

Investigators are actively testing interventions intended to increase lifespan [1]. Caloric restriction (CR) is the inter- vention most well established as able to increase lifespan in experimental models [2], and investigators are now seeking other interventions that may mimic the life-pro- 
longing effects of CR without requiring a reduction in caloric intake [3]. It is frequently said that CR not only increases average lifespan, but also 'maximum' lifespan [4]. Many researchers in the field of aging therefore wish to test whether other interventions increase maximum lifespan.

Recognizing this and the fact that one cannot be assured of observing population maximum lifespans in finite samples, Wang et al. [5] constructed and validated several tests (hereafter, the 'Wang-Allison tests') of differences in the upper parts of lifespan distributions by building on the work of Redden et al. [6] in the area of quantile regression. Wang et al. also showed that a commonly used test for differences in maximum lifespan that involved comparing the means of the top $p \%$ (e.g., top 10\%) of each of two samples (e.g., a treatment and a control sample) was not valid in that it had an excessive type-1 error rate. Nevertheless, there is appeal to using the full continuity of information in the upper tails of the sample distribution, and colleagues have recently suggested to us that a limitation of the Wang-Allison tests is that they only treat individual lifespans as being above or below some threshold defining 'old' or being in the tail of the survival distribution. That is, the Wang-Allison tests do not consider how much above the threshold any particular observation is, only whether the observation is above the threshold. We acknowledge this limitation and in response, we herein develop new tests that utilize the continuity of information among observations that exceed the threshold of interest, are more powerful than competing tests, including the Wang-Allison tests, in most cases, and remain valid under the null hypothesis of no effect on 'maximum' lifespan.

\section{Methods \\ Development of the tests}

Consider an experiment with two groups, treatment and control. The extension to more than two groups is straightforward (see discussion section). Let $X$ be an indicator variable taking the value 1 for observations in the treatment group and 0 for observations in the control group. Let $Y$ denote survival time. Let $\tau$ denote some threshold chosen by the investigator to denote an extreme portion of the distribution. In survival studies, $\tau$ can be chosen in advance to correspond to an age considered 'old' (e.g., 30 months in mice) or set to some high sample percentile (e.g., the 90th). Critically important, $\tau$ must be set to the same value for the two groups. That is, if $\tau$ is to be defined by an upper sample quantile, it should be the upper sample quantile of both of the two groups combined, not of each group separately.

Although not described in exactly these terms in the paper by Wang et al. [5], the Wang-Allison tests essentially create a new variable, $W$, where for the $\mathrm{i}^{\text {th }}$ subject, $W_{i} \equiv 0$ if $Y_{i} \leq \tau$, and $W_{i} \equiv 1$ if $Y_{i}>\tau$, and subsequently tests whether $W$ is associated with $X$ using an appropriate test statistic.

Thus, the Wang-Allison tests test the following null hypothesis:

$$
H_{0, A}: P(Y>\tau \mid X=1)=P(Y>\tau \mid X=0) .
$$

A problem with the Wang-Allison tests is that, hypothetically, $P(Y>\tau \mid X=1)$ may equal $P(Y>\tau \mid X=0)$ and yet the average magnitude by which lifespans exceed $\tau$ when $X=$ 1 may be radically different than when $\mathrm{X}=0$. This is exemplified in the hypothetical frequency distributions depicted in Figure 1. Note that these hypothetical distributions are not intended to be realistic, but only to clarify the point.

Let $X^{1}$ and $X^{0}$ denote the numbers of observations with $Y_{i}$ $>\tau$ in the treatment group and control group, respectively. The Wang-Allison tests use the test procedures for two independent binomial proportions [7] and these procedures require that $X^{1}$ and $X^{0}$ are independent. In the Wang-Allison tests, if the threshold is set in advance according to prior knowledge, $X^{1}$ and $X^{0}$ can satisfy the requirement of independence. But if $\tau$ is set to be the 90th percentile, $X^{1}$ and $X^{0}$ may not be independent, this creates a theoretical problem. However, on an empirical level, our simulations show that in the sample sizes we considered, this is not an apparent problem because the Wang-Allison tests have very high power and can control type I error quit well in the simulation studies and are
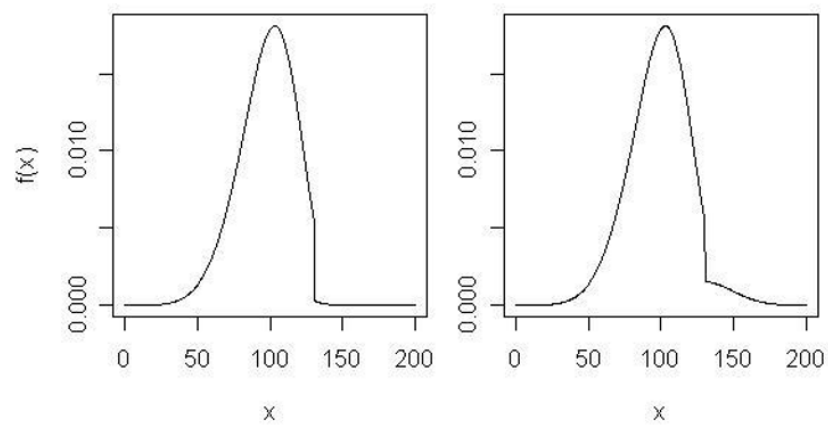

\section{Figure I}

The left graph is the density for control group $(X=0)$, $0.9 *$ Weibull $(5.73,106.6) * I(X \leq 130)+0.1 *$ Weibull $(5.40$, $100.06) *(X>130)$, and the right graph is the density for treatment group $(X=1), 0.9 *$ Weibull $(5.73,106.6) * 1(X \leq$ I30) + 0.I*Weibull $(5.45, \mid 30.06) * \mid(X>\mid 30)$, where $P(Y>$ $\tau \mid X=1)=P(Y>\tau \mid X=0)$ and yet the average magnitude by which lifespans exceed $\tau$ when $X=I$ is different than when $X$ $=0 . \tau$ is $90^{\text {th }}$ percentile of the all observations in treatment and control groups. 
practical for the lifespan studies). When $X^{1}$ and $X^{0}$ are not independent, simulation studies (including estimation of power and type I error) are an effective way to evaluate the methods (such as Wang-Allison tests) using the test procedures for two independent binomial proportions.

An alternative to testing $H_{0, A}$ is to test the following conceptually related but mathematically distinct null hypothesis:

$$
H_{0, B}: \mu(Y \mid Y>\tau \cap X=1)=\mu(Y \mid Y>\tau \cap X=0),
$$

where $\mu(\bullet)$ denotes the population mean (or expectation) of $(\bullet)$. Though appealing, a problem with testing $H_{0, B}$ is that when $P(Y>\tau \mid X=1)>P(Y>\tau \mid X=0)$ or $P(Y$ $>\tau \mid X=1)<<P(Y>\tau \mid X=0)$, for any finite sample with equal initial assignment to the two groups, $E\left[n_{0}\right]<<E\left[n_{1}\right]$ or $E\left[n_{0}\right] \gg E\left[n_{1}\right]$, where $E\left[n_{0}\right]$ denotes the expected number of observations in the control group for which $Y$ $>\tau$, and $E\left[n_{1}\right]$ denotes the expected number of observations in the treatment group for which $Y>\tau$. This imbalance between $E\left[n_{0}\right]$ and $E\left[n_{1}\right]$ will greatly reduce the power to reject $H_{0, B}$. In fact, in the extreme, when either $P$ $(Y>\tau \mid X=1)$ or $P(Y>\tau \mid X=0)$, there will be zero power to reject $H_{0, B}$ (actually, it is appropriate to say that $H_{0, B}$ is undefined in such cases). Such a situation is exemplified in the hypothetical frequency distributions depicted in Figure 2. Again, these hypothetical distributions are not intended to be realistic, but only to clarify the point.

Thus, one can conceive situations in which the power to reject $H_{0, A}$ will be zero and yet the upper tails of the distribution are clearly different. Similarly, one can conceive
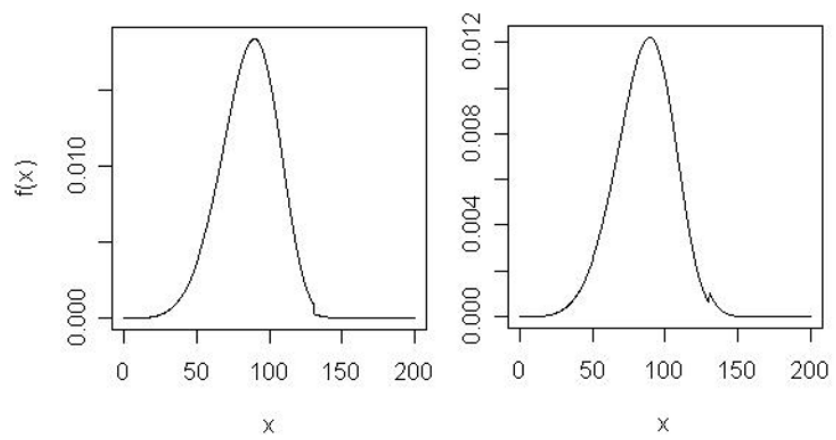

\section{Figure 2}

The left graph is the density for control group $(X=0)$, $0.9 *$ Weibull $(5.07,93.52) * I(X \leq 130)+0.1 *$ Weibull(5.40, $100.06) * \mathrm{I}(X>130)$, and the right graph for treatment group $(X=1), 0.6 *$ Weibull $(5.07,93.52) * \mathrm{I}(X \leq 130)+$

$0.4^{*}$ Weibull $(5.40,100.06) * \mathrm{l}(X>\mid 30)$, where $P(Y>\tau \mid X=1) \neq$ $P(Y>\tau \mid X=0), \mu(Y \mid Y>\tau \cap X=I)=\mu(Y \mid Y>\tau \cap X=0)$, and $\mu(\bullet)$ denotes the population mean of $(\bullet) . \tau$ is $90^{\text {th }}$ percentile of the all observations in treatment and control groups. situations in which the power to reject $H_{0, B}$ will be zero and yet again the upper tails of the distribution are clearly different. Hence, we propose a single-step union-intersection test [8] of the following compound null hypothesis:

$$
\begin{gathered}
H_{0, C}:[P(Y>\tau \mid X=1)=P(Y>\tau \mid X=0)] \cap[\mu(Y \mid Y>\tau \cap X= \\
1)=\mu(Y \mid Y>\tau \cap X=0)] .
\end{gathered}
$$

We construct the test of $H_{0, C}$ with the following simple procedure. Define a new variable $Z$ such that $Z_{i} \equiv I\left(Y_{i}>\right.$ $\tau) Y_{i}$, where $I(\bullet)$ denotes the indicator function taking on values of one if $(\bullet)$ is true and zero otherwise. One can then simply conduct an appropriate test (several candidates will be considered below) of whether the population mean of $\mathrm{Z}$ is significantly different between the treatment and control groups. This approach (hereafter new tests), has several desirable properties. First and foremost, when an appropriate test statistic is used, the approach will be valid. That is, unlike the conditional ttests (CTTs) commonly used and shown to be invalid by Wang et al. [5], when $H_{0, C}$ is true, it will only be rejected $100^{*} \alpha \%$ of the time at the nominal $\alpha$ level even if $f(Y \mid Y \leq$ $\tau \cap X=1) \neq f(Y \mid Y \leq \tau \cap X=0)$, where $f(\bullet)$ denotes the probability density function of $(\bullet)$.

Note that expectation (or population mean) of $Z, \mu(Z)=$ $P(Y>\tau) \mu(Y \mid Y>\tau)$. Therefore the new test for $H_{0, C}$ is really testing whether

$$
\begin{gathered}
P(Y>\tau \mid X=1) \mu(Y \mid Y>\tau \cap X=1)=P(Y>\tau \mid X=0) \mu \\
(Y \mid Y>\tau \cap X=0),
\end{gathered}
$$

while the method for $H_{0, B}$ is testing whether $\mu(Y \mid Y>\tau \cap$ $X=1)=\mu(Y \mid Y>\tau \cap X=0)$ and the method for $H_{0, A}$ is testing whether $P(Y>\tau \mid X=1)=P(Y>\tau \mid X=0)$. The mean difference of $\mu(Z)$ between two groups consists of two components: the difference between probabilities $P(Y$ $>\tau \mid X=1)$ and $P(Y>\tau \mid X=0)$ and the difference between expectations $\mu(Y \mid Y>\tau \cap X=1)$ and $\mu(Y \mid Y>\tau \cap X=$ $0)$. The test for $H_{0, A}$ focuses on the first component and the test for $H_{0, A}$ focuses on the second one, while the test for $H_{0, C}$ is related to both components.

We also note that Dominici and Zeger [9] studied similar mean difference components for two groups (cases and controls) by estimating the mean difference $\Delta(v)$ for the two groups conditional on a vector of covariates $v$ for zero-inflated data through smooth quantile ratio estimation with regression,

$$
\begin{gathered}
\Delta(v)=P(Y>0 \mid X=1, v) \mu(Y \mid Y>0, X=1, v)-P(Y>0 \mid X \\
=0, v) \mu(Y \mid Y>0, X=0, v),
\end{gathered}
$$

where, $Y$ is nonnegative random variable denoting the health expenditures. While Dominici and Zeger [9] esti- 
mate the mean difference of nonnegative random variables $(Y)$ for two groups, our methods test the mean difference of random variables $(Y)$ which are greater than threshold $\tau$.

\section{Evaluation of the tests}

We evaluate the tests via computer simulation. For each scenario simulated, we evaluate the tests at the 2-tailed 05 $\alpha$ level and at the 2-tailed .01 $\alpha$ level using 5,000 simulated datasets per scenario (except for permutation tests where we use 1,000 datasets per scenario and 1,000 random permutations by Monte Carlo sampling for each dataset). In simulation 1, we first evaluate performance in simulation under the null hypothesis $H_{0, C}$ (i.e., both $H_{0, A}$ and $H_{0, B}$ are true) and yet $f(Y \mid Y \leq \tau \cap X=1)$ is radically different from $f(Y \mid Y \leq \tau \cap X=0)$. After showing that the tests remain valid even in these extreme circumstances, we compare their power in several scenarios (simulations 2-4) described below. For each scenario, we assumed that there were two groups with an equal number of subjects per group. We ran scenarios with 50,80 , or 100 subjects in each of the two groups, realistic sample sizes for animal model longevity research.
We simulated data using a concatenation of Weibull distributions to flexibly emulate the data observed in a real study [10] of obese animals (control; $\mathrm{X}=0$ ) versus animals that were obese and then lost weight via CR (treatment; $X=1)$. Specifically, For example, in simulations 1-4, we simulated $Y$ from the following distribution:

$f(Y \mid X=j)=r_{j}\left[\frac{b_{j, L}}{a_{j, L}}\left(\frac{Y}{a_{j, L}}\right)^{b_{j, L}-1} e^{-\left(\frac{Y}{a_{j, L}}\right)^{b_{j, L}}}\right] I(Y \leq 130)+\left(1-r_{j}\right)\left[\frac{b_{j, U}}{a_{j, U}}\left(\frac{Y}{a_{j, U}}\right)^{b_{j, u}-1} e^{-\left(\frac{Y}{a_{j, U}}\right)^{b_{j, U}}}\right] I(Y>130)$, where $\mathrm{j}=0$ to 1 , lifespan $(\mathrm{Y})$ is measured in weeks, $\mathrm{a}_{\mathrm{i}, \mathrm{L}}$ and $b_{i, L}$ are the parameters of a Weibull distribution for the lower $90 \%$ of the distribution, and $\mathrm{a}_{\mathrm{j}, \mathrm{u}}$ and $\mathrm{b}_{\mathrm{i}, \mathrm{u}}$ are the parameters of a Weibull distribution for the upper $10 \%$ of the distribution. $r_{j}$ is a proportion parameter, for example $r_{j}=0.9$. The specific values of the parameters used are provided in Figure 3.

\section{Delineation of tests to be evaluated}

Each of the tests listed below was implemented in two manners, first with $\tau$ set in advance to a fixed lifespan value (130 weeks), and second with $\tau$ set at the sample $90^{\text {th }}$ percentile of the two groups combined. In real-life situations, one usually does know the threshold of interest $a$ priori. We do recognize that we will not have such knowl-

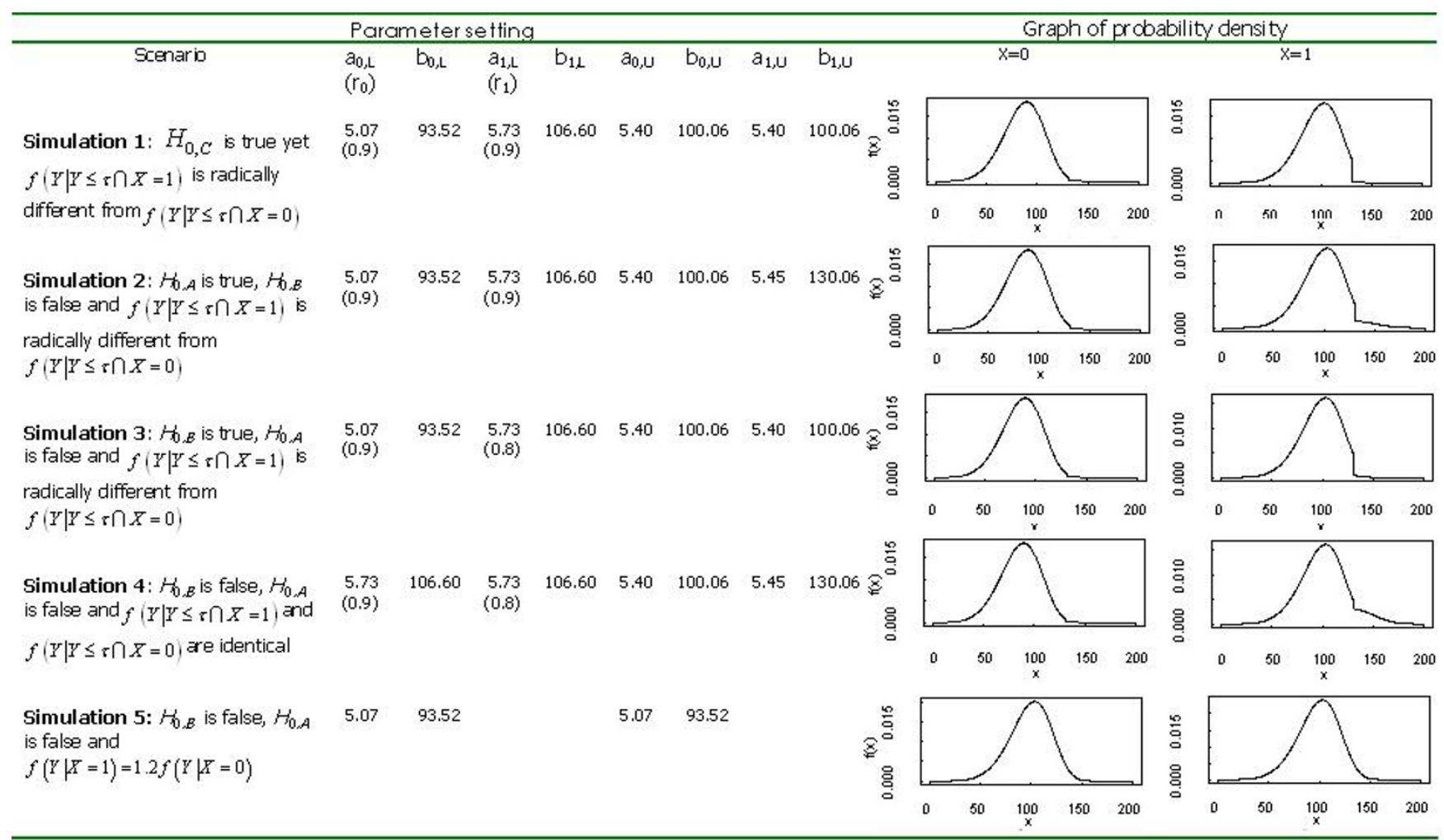

Figure 3

Parameter values and distributions for component Weibull distributions used in each simulation. 
edge in all cases. It is for this reason that when analyzing the simulated data, we also consider a threshold of the $90^{\text {th }}$ percentile of the data allowing for an ad hoc databased determination of a threshold.

\section{Tests of $\mathrm{H}_{0, A}$ (Wang-Allison tests)}

For comparative purposes, the first category of tests we evaluated were the tests denoted QT3 and QT4 in Wang et al [5] which are, respectively, Boschloo's test and an exact unconditional test based on the observed difference divided by its estimated standard error under the null hypothesis (score statistic) and are described in more detail by Mehrotra et al. [7]. These were the two tests that Wang et al. [5] had found performed best as tests of $H_{0, A}$.

\section{Tests of $\mathrm{H}_{0, B}$}

In testing $H_{0, B}$, subjects were only included in the analysis when their lifespans exceeded $\tau$. Distributions of survival times (lifespans) are rarely Gaussian and, even if they were nearly Gaussian after, for example, log transformation, the distribution of just the tail portion (i.e., $f(Y \mid Y>$ $\tau)$ would not be. Hence, in constructing tests we relied on nonparametric statistical methods. Specifically, we used the Wilcoxon-Mann-Whitney (exact) test $[11,12]$ and a permutation test (with t-statistic) as described by Good [13] to test for differences in lifespan among those subjects whose lifespans exceeded $\tau$.

\section{Tests of $\mathrm{H}_{0, \mathrm{C}}$ (new tests)}

In testing $H_{0, C}$ all subjects were analyzed, but the variable analyzed was $Z$ as defined above. Because the distribution of $Z$ cannot be normally distributed, we again used the Wilcoxon-Mann-Whitney test and a permutation test to test for differences in $\mathrm{Z}$.

For a dataset with $n_{1}\left(n_{2}\right)$ subjects in treatment (control) group, the permutation test can be performed in the following way: First put all the $\left(n_{1}+n_{2}\right)$ subjects together, and then generate 1000 replicated datasets. For each replicated dataset, we randomly sample $n_{1}$ subjects from the $\left(n_{1}+n_{2}\right)$ subjects and assign them to treatment group, and assign the left $n_{2}$ subjects to control group. We run T-test on the observed dataset and the 1000 replicated datasets. Let $T_{0}$ be the $\mathrm{T}$ value for the observed dataset, then $\mathrm{p}$-value for the permutation test is calculated as the proportion of replicated datasets with absolute $\mathrm{T}$ values greater than or equal to the absolute valued of $T_{0}$.

\section{Results}

Results are displayed in Tables 1 to 5 . As can be seen, the new methods for tests of $H_{0, C}$ controls type I error rates quite well. The power of the new methods are always higher than or very close to that of the methods for tests of $H_{0, A}$ (Wang-Allison tests) and are higher than that of the methods for tests of $H_{0, B}$ (Wilcoxon-Mann-Whitney tests and permutation tests for observations above the threshold $\tau$ ) in some of the simulations.

Table 1 shows the type I error rate of the tests (in simulation 1) when the null hypothesis $\mathrm{HO}, \mathrm{C}$ is true (i.e., both $\mathrm{HO}, \mathrm{A}$ and $\mathrm{HO}, \mathrm{B}$ are true) and yet $\mathrm{f}(\mathrm{Y} \mid \mathrm{Y} \leq \tau \cap \mathrm{X}=1)$ is radically differentfrom $f(Y \mid Y \leq \tau \cap X=0)$. The type I error rates of the new methods are comparable to those of the methods for tests of H0,A and those of the methods for tests of HO,B . It is note worthy that there is a slight but fairly consistent excess of type I errors when the sample 90th percentile is used rather than a fixed cutoff point. This is because the sample 90th percentile is a random variable and when it falls below its population level, the null hypothesis is no longer strictly true in our simulations. That is, the tests remain valid tests of differences in distributions above the actual value used but should not be strictly interpreted as tests of differences in distributions above the 90th (or any other percentile). In practice, this distinction is probably trivial.

In simulation 2 (see Table 2), where $H_{0, A}$ is true, $H_{0, B}$ is false and $f(Y \mid Y \leq \tau \cap X=1)$ is radically different from $f$ $(Y \mid Y \leq \tau \cap X=0)$, the new methods for tests of $H_{0, C}$ and the methods for tests of $H_{0, A}$ have lower power than that of the corresponding methods for tests of $H_{0, B}$, however, the new methods for tests of $H_{0, C}$ can slightly improve the power compared to the methods for tests of $H_{0, A}$.

Table 3 shows the power of the tests in Simulation 3, where $H_{0, B}$ is true, $H_{0, A}$ is false and $f(Y \mid Y \leq \tau \cap X=1)$ is radically different from $f(Y \mid Y \leq \tau \cap X=0)$. The new methods for tests of $H_{0, C}$ and the methods for tests of $H_{0, A}$ have very similar power which is much higher than that of the corresponding methods for tests of $H_{0, B}$.

From simulation 4 (see Table 4), where $H_{0, B}$ is false, $H_{0, A}$ is false and $f(Y \mid Y \leq \tau \cap X=1)$ and $f(Y \mid Y \leq \tau \cap X=0)$ are identical, we can find that the new methods for tests of $H_{0, C}$ always have higher power than the corresponding methods for tests of $H_{0, A}$. When $\tau$ being set to the 90th percentile of the sample, the new methods also have higher power than the corresponding methods for tests of $H_{0, B}$.

Finally, we conducted a set of simulations under what we perceived to be the most realistic situations. Here both $H_{0, A}$ and $H_{0, B}$ are false, $f(Y \mid Y \leq \tau \cap X=1)$ is quite different from $f(Y \mid Y \leq \tau \cap X=0)$, and the distributions have no discontinuities. In other words, there is just a simple reduction in the hazard rate when $X=1$. Table 5 presents the power of the tests in Simulation 5, where $f(Y \mid X=1)=1.2 f$ $(Y \mid X=0)$. In this simulation, the methods for tests of $H_{0, B}$ 
Table I: Performance (type I error rates) of the tests in simulation I under $\boldsymbol{H}_{0, C}$ (i.e., both $\boldsymbol{H}_{0, A}$ and $\boldsymbol{H}_{0, B}$ are true) and yet $f(Y \mid Y \leq \tau \cap X$ $=I$ ) is radically different from $f(Y \mid Y \leq \tau \cap X=0)$ (see Figure 3 for details of simulation).

\begin{tabular}{|c|c|c|c|c|c|c|}
\hline \multirow[t]{3}{*}{ Test } & \multicolumn{6}{|c|}{ Sample Size (N) Per Group } \\
\hline & \multicolumn{2}{|c|}{50} & \multicolumn{2}{|c|}{80} & \multicolumn{2}{|c|}{100} \\
\hline & $\alpha=.05$ & $\alpha=.01$ & $\alpha=.05$ & $\alpha=.01$ & $\alpha=.05$ & $\alpha=.01$ \\
\hline \multicolumn{7}{|c|}{ Tests of $H_{0, A}$ (Wang-Allison tests) } \\
\hline $\begin{array}{l}\text { QT3 with } \tau \text { set to } \\
\text { I30. }\end{array}$ & $0.032(.027, .036)^{\#}$ & $0.008(.005, .011)$ & $0.041(.036, .046)$ & $0.006(.003, .009)$ & $0.040(.035, .045)$ & $0.006(.003, .009)$ \\
\hline $\begin{array}{l}\text { QT3 with } \tau \text { set to } \\
\text { sample } 90^{\text {th }} \text { percentile }\end{array}$ & $0.026(.022, .030)$ & $0.026 *(.020, .032)$ & $0.080(.072, .088)$ & $0.007(.004, .010)$ & $0.040(.035, .045)$ & $0.010(.006, .014)$ \\
\hline $\begin{array}{l}\text { QT4 with } \tau \text { set to } \\
\text { I30. }\end{array}$ & $0.038(.033, .043)$ & $0.008(.005, .011)$ & $0.051(.045, .057)$ & $0.009(.006, .012)$ & $0.047(.041, .053)$ & $0.007(.004, .010)$ \\
\hline $\begin{array}{l}\text { QT4 with } \tau \text { set to } \\
\text { sample } 90^{\text {th }} \text { percentile. }\end{array}$ & $0.026(.022, .030)$ & $0.026(.020, .032)$ & $0.083(.075, .091)$ & $0.026(.020, .032)$ & $0.040(.035, .045)$ & $0.010(.006, .014)$ \\
\hline \multicolumn{7}{|l|}{ Tests of $H_{0, B}$} \\
\hline $\begin{array}{l}\text { Wilcoxon-Mann- } \\
\text { Whitney** with } \tau \text { set } \\
\text { to I } 30 \text {. }\end{array}$ & $0.04 I(.036, .046)$ & $0.017(.012, .022)$ & $0.044(.038, .050)$ & $0.008(.005, .011)$ & $0.046(.040, .052)$ & $0.008(.005, .011)$ \\
\hline $\begin{array}{l}\text { Wilcoxon-Mann- } \\
\text { Whitney with } \tau \text { set to } \\
\text { sample } 90^{\text {th }} \text { percentile. }\end{array}$ & $0.049(.043, .055)$ & $0.014(.010, .018)$ & $0.065(.058, .072)$ & $0.015(.011, .019)$ & $0.080(.072, .088)$ & $0.018(.013, .023)$ \\
\hline $\begin{array}{l}\text { Permutation test with } \\
\tau \text { set to } 130 \text {. }\end{array}$ & $0.050(.036, .064)$ & $0.009(.001, .017)$ & $0.050(.036, .064)$ & $0.011(.002, .020)$ & $0.064(.049, .079)$ & $0.015(.005, .025)$ \\
\hline $\begin{array}{l}\text { Permutation test with } \\
\tau \text { set to sample } 90^{\text {th }} \\
\text { percentile. }\end{array}$ & $0.077(.060, .094)$ & $0.016(.006, .026)$ & $0.078(.06 \mathrm{I}, .095)$ & $0.022(.010, .034)$ & $0.083(.066, .100)$ & $0.019(.008, .030)$ \\
\hline \multicolumn{7}{|c|}{ Tests of $H_{0, c}$ (new tests) } \\
\hline $\begin{array}{l}\text { Wilcoxon-Mann- } \\
\text { Whitney with } \tau \text { set to } \\
\text { 130. }\end{array}$ & $0.042(.036, .048)$ & $0.007(.004, .010)$ & $0.049(.043, .055)$ & $0.010(.006, .014)$ & $0.051(.045, .057)$ & $0.008(.005, .011)$ \\
\hline $\begin{array}{l}\text { Wilcoxon-Mann- } \\
\text { Whitney with } \tau \text { set to } \\
\text { sample } 90^{\text {th }} \text { percentile. }\end{array}$ & $0.055(.049, .061)$ & $0.015(.011, .019)$ & $\mathbf{0 . 0 6 0}(.053, .067)$ & $0.015(.011, .019)$ & $0.061(.054, .068)$ & $0.015(.011, .019)$ \\
\hline $\begin{array}{l}\text { Permutation test with } \\
\tau \text { set to } 130 \text {. }\end{array}$ & $0.052(.038, .066)$ & $0.015(.005, .025)$ & $0.047(.034, .060)$ & $0.009(.001, .017)$ & $0.057(.043, .07 I)$ & $0.007(.000, .014)$ \\
\hline $\begin{array}{l}\text { Permutation test with } \\
\tau \text { set to sample } 90^{\text {th }} \\
\text { percentile. }\end{array}$ & $0.045(.032, .058)$ & $0.017(.006, .028)$ & $0.062(.047, .077)$ & $0.011(.002, .020)$ & $0.068(.053, .084)$ & $0.018(.007, .029)$ \\
\hline
\end{tabular}

\#2-tailed $95 \%$ confidence interval.

*The bolded values are those simulated type I error rates which are significantly higher than the nominal $\alpha$ at the 2 -tailed $95 \%$ confidence level (i.e., the lower bound of the interval is higher than $\alpha$ ). Note that for the permutation tests we used 1000 replicated datasets and for other tests we used 5000 replicated datasets.

**In all the simulation studies (Tables I-5), we used Wilcoxon-Mann-Whitney exact test.

almost have no power because the control group always has no or few observations above the threshold $\tau$. The new methods for tests of $H_{0, C}$, when using a permutation test, have power higher than or equal to that of the methods for tests of $H_{0, A}$.

\section{Illustration with real data}

To illustrate the methods, we applied them to two real datasets. In both of these datasets, prior research had shown differences in overall survival rate and we tested for differences in 'maximum lifespan' herein. The first was a subset of data reported by Vasselli et al [10]. The subset of the data consists of two groups of Sprague-Dawley rats, those kept on a high-fat diet ad libitum throughout life and becoming obese (EO-HF) and those kept on a highfat diet ad libitum until early-middle adulthood, becoming obese, and subsequently reduced to normal weight via caloric restriction, but on the same high-fat diet (WL-HF). Each group had 49 rats (see Figure 4 for the histograms for the data). The second dataset was from a study comparing the lifespan of Agouti-related protein-deficient (AgRP(-/)) mice to wildtype mice $(+/+)$ as reported by Redmann \& Argyropoulos [14]. This dataset consists of 16 mice with genotype '+/+' and 21 mice with genotype '-/-' (see Figure 5 for the histograms for this dataset). From Figure 4, we 
Table 2: Performance of the tests in simulation 2, $H_{0, A}$ is true, $H_{0, B}$ is false and $f(Y \mid Y \leq \tau \cap X=I)$ is radically different from $f(Y \mid Y \leq \tau \cap X$ $=0$ ) (see Figure 3 for details of simulation).

\begin{tabular}{|c|c|c|c|c|c|c|}
\hline \multirow[t]{3}{*}{ Test } & \multicolumn{6}{|c|}{ Sample Size (N) Per Group } \\
\hline & \multicolumn{2}{|c|}{50} & \multicolumn{2}{|c|}{80} & \multicolumn{2}{|c|}{100} \\
\hline & $\alpha=.05$ & $\alpha=.01$ & $\alpha=.05$ & $\alpha=.01$ & $\alpha=.05$ & $\alpha=.01$ \\
\hline \multicolumn{7}{|l|}{ Tests of $H_{0, A}$ (Wang-Allison tests) } \\
\hline QT3 with $\tau$ set to 130 & 0.032 & 0.008 & 0.041 & 0.006 & 0.040 & 0.006 \\
\hline QT3 with $\tau$ set to sample $90^{\text {th }}$ percentile. & 0.034 & 0.034 & 0.104 & 0.009 & 0.062 & 0.018 \\
\hline QT4 with $\tau$ set to 130 . & 0.038 & 0.008 & 0.051 & 0.009 & 0.047 & 0.007 \\
\hline QT4 with $\tau$ set to sample $90^{\text {th }}$ percentile. & 0.034 & 0.034 & 0.104 & 0.033 & 0.062 & 0.018 \\
\hline \multicolumn{7}{|l|}{ Tests of $H_{0, B}$} \\
\hline Wilcoxon-Mann-Whitney with $\tau$ set to 130 & 0.264 & 0.090 & 0.504 & 0.261 & 0.631 & 0.368 \\
\hline Wilcoxon-Mann-Whitney with $\tau$ set to sample $90^{\text {th }}$ percentile. & 0.16 & 0.051 & 0.314 & 0.143 & 0.406 & 0.220 \\
\hline Permutation test with $\tau$ set to 130 & 0.337 & 0.111 & 0.608 & 0.332 & 0.737 & 0.456 \\
\hline Permutation test with $\tau$ set to sample $90^{\text {th }}$ percentile. & 0.197 & 0.047 & 0.423 & 0.204 & 0.525 & 0.284 \\
\hline \multicolumn{7}{|l|}{ Tests of $H_{0, c}$ (new tests) } \\
\hline Wilcoxon-Mann-Whitney with $\tau$ set to 130 . & 0.051 & 0.008 & 0.062 & 0.012 & 0.056 & 0.010 \\
\hline Wilcoxon-Mann-Whitney with $\tau$ set to sample $90^{\text {th }}$ percentile. & 0.107 & 0.029 & 0.090 & 0.028 & 0.124 & 0.035 \\
\hline Permutation test with $\tau$ set to 130 . & 0.061 & 0.013 & 0.055 & 0.012 & 0.065 & 0.014 \\
\hline Permutation test with $\tau$ set to sample $90^{\text {th }}$ percentile. & 0.109 & 0.032 & 0.097 & 0.03 & 0.129 & 0.046 \\
\hline
\end{tabular}

can see the upper tails of the histograms of the two groups are different. Similar results can be found in Figure 5.

Results ( $\mathrm{p}$ values of tests) are shown in Table 6. As can be seen, when setting $\tau$ equal to 110 (100) for the first (second) datasets, both the methods for tests of $H_{0, A}$ and the new methods for tests of $H_{0, C}$ can detect the differences in 'maximum lifespan' between groups at nominal alpha levels of $0.01(0.05)$ for the first (second) datasets. But the methods for tests of $H_{0, B}$ cannot detect the difference for all different values of $\tau$. The following description may provide some explanation to these results. For the first dataset, when set $\tau=110$, the proportions of the observations greater than $\tau$ in the EO-HF group and WL-HF group (i.e., estimations of $P(Y>\tau \mid X=0)$ and $P(Y>\tau \mid X=1)$ ) are 0.061 and 0.306 , respectively. These two proportions are significantly different and not surprisingly, the methods for tests of $H_{0, A}$ can detect the difference in 'maximum

Table 3: Performance of the tests in simulation 3, $H_{0, B}$ is true, $H_{0, A}$ is false and $f(Y \mid Y \leq \tau \cap X=I)$ is radically different from $f(Y \mid Y \leq \tau \cap X$ $=0$ ) (see Figure 3 for details of simulation).

Test Sample Size (N) Per Group

\begin{tabular}{|c|c|c|c|c|c|}
\hline \multicolumn{2}{|c|}{50} & \multicolumn{2}{|c|}{80} & \multicolumn{2}{|c|}{100} \\
\hline$\alpha=.05$ & $\alpha=.01$ & $\alpha=.05$ & $\alpha=.01$ & $\alpha=.05$ & $\alpha=.01$ \\
\hline
\end{tabular}

Tests of $\mathrm{H}_{0, A}$ (Wang-Allison tests)

QT3 with $\tau$ set to 130 .

QT3 with $\tau$ set to sample $90^{\text {th }}$ percentile.

QT4 with $\tau$ set to 130 .

QT4 with $\tau$ set to sample $90^{\text {th }}$ percentile.

Tests of $\mathrm{H}_{0, B}$

Wilcoxon-Mann-Whitney with $\tau$ set to 130 .

Wilcoxon-Mann-Whitney with $\tau$ set to sample $90^{\text {th }}$ percentile.

Permutation test with $\tau$ set to 130 .

Permutation test with $\tau$ set to sample $90^{\text {th }}$ percentile.

Tests of $H_{0, c}$ (new tests)

Wilcoxon-Mann-Whitney with $\tau$ set to 130 .

Wilcoxon-Mann-Whitney with $\tau$ set to sample $90^{\text {th }}$ percentile.

Permutation test with $\tau$ set to 130 .

Permutation test with $\tau$ set to sample $90^{\text {th }}$ percentile.

$\begin{array}{llllll}0.244 & 0.101 & 0.412 & 0.181 & 0.490 & 0.258 \\ 0.102 & 0.102 & 0.332 & 0.051 & 0.297 & 0.143 \\ 0.266 & 0.102 & 0.418 & 0.187 & 0.514 & 0.274 \\ 0.102 & 0.102 & 0.332 & 0.151 & 0.297 & 0.143 \\ & & & & & \\ 0.046 & 0.013 & 0.049 & 0.011 & 0.045 & 0.008 \\ 0.048 & 0.019 & 0.044 & 0.01 & 0.041 & 0.009 \\ 0.042 & 0.007 & 0.046 & 0.012 & 0.064 & 0.013 \\ 0.046 & 0.009 & 0.046 & 0.012 & 0.044 & 0.01 \\ & & & & & \\ 0.276 & 0.111 & 0.420 & 0.201 & 0.517 & 0.271 \\ 0.182 & 0.07 & 0.278 & 0.104 & 0.35 & 0.154 \\ 0.291 & 0.101 & 0.427 & 0.203 & 0.515 & 0.28 \\ 0.169 & 0.067 & 0.264 & 0.107 & 0.363 & 0.173\end{array}$


Table 4: Performance of the tests in simulation 4, $H_{0, B}$ is false, $H_{0, A}$ is false and $f(Y \mid Y \leq \tau \cap X=I)$ and $f(Y \mid Y \leq \tau \cap X=0)$ are identical (see Figure 3 for details of simulation).

\begin{tabular}{|c|c|c|c|c|c|c|}
\hline \multirow[t]{3}{*}{ Test } & \multicolumn{6}{|c|}{ Sample Size (N) Per Group } \\
\hline & \multicolumn{2}{|c|}{50} & \multicolumn{2}{|c|}{80} & \multicolumn{2}{|c|}{100} \\
\hline & $\alpha=.05$ & $\alpha=.01$ & $\alpha=.05$ & $\alpha=.01$ & $\alpha=.05$ & $\alpha=.01$ \\
\hline \multicolumn{7}{|l|}{ Tests of $H_{0, A}$ (Wang-Allison tests) } \\
\hline QT3 with $\tau$ set to 130 & 0.244 & 0.101 & 0.412 & 0.181 & 0.490 & 0.258 \\
\hline QT3 with $\tau$ set to sample $90^{\text {th }}$ percentile. & 0.363 & 0.363 & 0.735 & 0.337 & 0.753 & 0.600 \\
\hline QT4 with $\tau$ set to 130 . & 0.266 & 0.102 & 0.418 & 0.187 & 0.514 & 0.274 \\
\hline QT4 with $\tau$ set to sample $90^{\text {th }}$ percentile. & 0.363 & 0.363 & 0.735 & 0.555 & 0.753 & 0.600 \\
\hline \multicolumn{7}{|l|}{ Tests of $H_{0, B}$} \\
\hline Wilcoxon-Mann-Whitney with $\tau$ set to 130 . & 0.409 & 0.172 & 0.684 & $0.4 I I$ & 0.804 & 0.56 \\
\hline Wilcoxon-Mann-Whitney with $\tau$ set to sample $90^{\text {th }}$ percentile. & 0.245 & 0.142 & 0.33 & 0.144 & 0.434 & 0.176 \\
\hline Permutation test with $\tau$ set to 130 . & 0.517 & 0.244 & 0.81 & 0.568 & 0.913 & 0.728 \\
\hline Permutation test with $\tau$ set to sample $90^{\text {th }}$ percentile. & 0.169 & 0.039 & 0.428 & 0.190 & 0.569 & 0.249 \\
\hline \multicolumn{7}{|l|}{ Tests of $H_{0, c}$ (new tests) } \\
\hline Wilcoxon-Mann-Whitney with $\tau$ set to 130 . & 0.374 & $0.17 \mid$ & 0.528 & 0.280 & 0.629 & 0.373 \\
\hline Wilcoxon-Mann-Whitney with $\tau$ set to sample $90^{\text {th }}$ percentile. & 0.602 & 0.353 & 0.734 & 0.552 & 0.865 & 0.724 \\
\hline Permutation test with $\tau$ set to 130 . & 0.393 & 0.177 & 0.524 & 0.288 & 0.626 & 0.377 \\
\hline Permutation test with $\tau$ set to sample $90^{\text {th }}$ percentile. & 0.619 & 0.365 & 0.726 & 0.553 & 0.852 & 0.704 \\
\hline
\end{tabular}

lifespan' between the two groups. Second, the sample means of the observations greater than $\tau$ in the two groups (i.e., estimations of $\mu(Y \mid Y>\tau \cap X=1)$ and $\mu(Y \mid Y>\tau$ $\cap X=0)$ ) are 117.8 and 122.9 , respectively, and there is no much difference between these sample means. However the sample means of the $Z$-values in the two group (i.e., the estimations of $P(Z \mid X=0)$ and $P(Z \mid X=1)$ ) are 7.210 and 37.633, respectively, and are greatly different, where, $Z_{i} \equiv I\left(Y_{i}>\tau\right) Y_{i}$. These may explain that the methods for tests of $H_{0, B}$ cannot reject the null but the new methods for tests of $H_{0, C}$ can detect the difference in 'maximum lifespan' between the two groups. Similarly, for the second dataset, when set $\tau=100$, the proportions of the observations greater than $\tau$ in the group with genotype '+/ +' and group with genotype '-/-' are 0.188 and 0.571 , respectively. The sample means of the observations greater than $\tau$ in the two groups are 109.3 and 110.9,

Table 5: Performance of the tests in simulation $5, H_{0, B}$ is false, $H_{0, A}$ is false and $f(Y \mid X=I)=1.2 f(Y \mid X=0)$ (see Figure 3 for details of simulation).

Test Sample Size (N) Per Group

\begin{tabular}{cccccc}
\hline \multicolumn{2}{c}{50} & \multicolumn{2}{c}{80} & \multicolumn{2}{c}{100} \\
\cline { 3 - 5 } & $\alpha=.05$ & $\alpha=.05$ & $\alpha=.01$
\end{tabular}

Tests of $H_{0, A}$ (Wang-Allison tests)

QT3 with $\tau$ set to 130 .

QT3 with $\tau$ set to sample $90^{\text {th }}$ percentile.

QT4 with $\tau$ set to 130 .

QT4 with $\tau$ set to sample $90^{\text {th }}$ percentile.

Tests of $\mathrm{H}_{0, B}$

Wilcoxon-Mann-Whitney with $\tau$ set to 130 .

Wilcoxon-Mann-Whitney with $\tau$ set to sample $90^{\text {th }}$ percentile.

Permutation test with $\tau$ set to 130

Permutation test with $\tau$ set to sample $90^{\text {th }}$ percentile.

0.663

0.815

0.765

0.815

0.349

0.815

0.349

0.815

0.001

0.016

0.001

0.032

Tests of $\mathrm{H}_{0, \mathrm{c}}$ (new tests)

Wilcoxon-Mann-Whitney with $\tau$ set to 130 .

Wilcoxon-Mann-Whitney with $\tau$ set to sample $90^{\text {th }}$ percentile.

Permutation test with $\tau$ set to 130 .

Permutation test with $\tau$ set to sample $90^{\text {th }}$ percentile.

$\begin{array}{llll}0.925 & 0.754 & 0.965 & 0.883 \\ 0.996 & 0.885 & 0.997 & 0.986 \\ 0.941 & 0.797 & 0.981 & 0.906 \\ 0.996 & 0.969 & 0.997 & 0.986 \\ & & & \\ 0.006 & 0.000 & 0.010 & 0.000 \\ 0.035 & 0.002 & 0.058 & 0.009 \\ 0.036 & 0.003 & 0.061 & 0.005 \\ 0.082 & 0.017 & 0.124 & 0.041 \\ & & & \\ 0.920 & 0.742 & 0.979 & 0.897 \\ 0.995 & 0.964 & 0.999 & 0.992 \\ 0.960 & 0.850 & 0.993 & 0.940 \\ 0.995 & 0.958 & 0.997 & 0.986\end{array}$



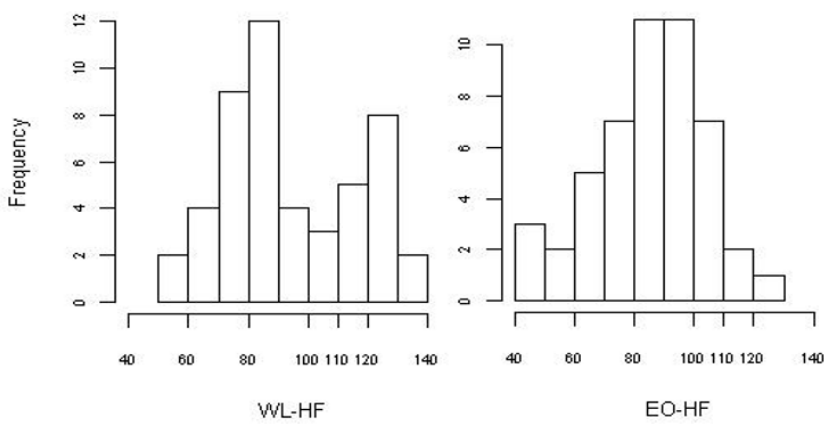

Figure 4

The left (right) graph is the histogram of lifespan for WL-HF (EO-HF) group in the data from Vasselli et al. [10].

respectively. The sample means of the Z-values in the two groups are 20.5 and 63.4 respectively.

From Table 6 we can also see that in almost all situations the p-values of the new methods for tests of $H_{0, C}$ are somewhat smaller than those of the methods for tests of $H_{0, A}$. This is consistent with the simulations showing greater power of the new methods.

\section{Discussion}

Herein, we proposed new methods for testing the difference of 'maximum' lifespan between groups (e.g., treatment and control). By defining a new variable $Z$ such that $Z_{i} \equiv I\left(Y_{i}>\tau\right) Y_{i}$ for each observation and then applying
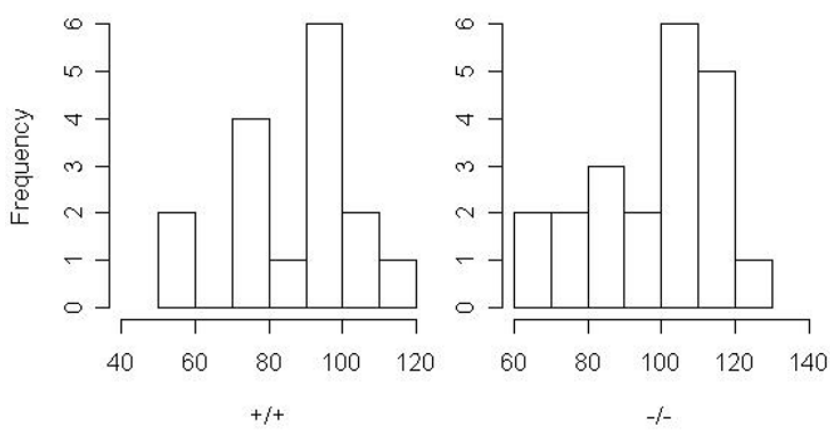

\section{Figure 5}

The left (right) graph is the histogram of lifespan for group with genotype '+/+' ('-/-') in the data from Redmann \& Argyropoulos [14].

Wilcoxon-Mann-Whitney test or better still a permutation test to $Z$, the new methods achieve far better performance when considered across a broad range of circumstances in terms of both Type-1 error rates and power. In the new methods, we use the Wilcoxon-Mann-Whitney test or permutation test. One could also choose to use a bootstrap test in place of these two tests. However, additional simulations would likely be warranted to evaluate its performance relative to the permutation test we have evaluated herein.

It is straightforward to extend the new methods to more than two groups. For example, one could use the KruskalWallis Test to replace the Wilcoxon-Mann-Whitney test, or

Table 6: Results (p values of tests) of application to two real datasets.

\begin{tabular}{|c|c|c|}
\hline Test & Data from Vasselli et al. [10]' & Data from Redmann \& Argyropoulos [14] ${ }^{2}$ \\
\hline \multicolumn{3}{|l|}{ Tests of $H_{0, A}$ (Wang-Allison tests) } \\
\hline QT3 with $\tau$ set to $110 / 100 \#$ & 0.002 & 0.027 \\
\hline QT3 with $\tau$ set to sample $90^{\text {th }}$ percentile. & 0.038 & 0.186 \\
\hline QT4 with $\tau$ set to $110 / 100$ & 0.002 & 0.022 \\
\hline QT4 with $\tau$ set to sample $90^{\text {th }}$ percentile. & 0.033 & 0.146 \\
\hline \multicolumn{3}{|l|}{ Tests of $H_{0, B}$} \\
\hline Wilcoxon-Mann-Whitney with $\tau$ set to $110 / 100$. & 0.289 & 0.868 \\
\hline $\begin{array}{l}\text { Wilcoxon-Mann-Whitney with } \tau \text { set to sample } 90^{\text {th }} \\
\text { percentile. }\end{array}$ & 0.750 & $\mathbf{N} / \mathbf{A}^{*}$ \\
\hline Permutation test with $\tau$ set to $110 / 100$ & 0.281 & 0.738 \\
\hline Permutation test with $\tau$ set to sample $90^{\text {th }}$ percentile. & 0.634 & $\mathbf{N} / \mathbf{A} *$ \\
\hline \multicolumn{3}{|l|}{ Tests of $H_{0, c}$ (new tests) } \\
\hline Wilcoxon-Mann-Whitney with $\tau$ set to $110 / 100$ & 0.001 & 0.022 \\
\hline $\begin{array}{l}\text { Wilcoxon-Mann-Whitney with } \tau \text { set to sample } 90^{\text {th }} \\
\text { percentile. }\end{array}$ & 0.026 & 0.243 \\
\hline Permutation test with $\tau$ set to $110 / 100$. & 0.001 & 0.014 \\
\hline Permutation test with $\tau$ set to sample $90^{\text {th }}$ percentile. & 0.024 & 0.072 \\
\hline
\end{tabular}

Notes: In each dataset, males and females have been combined. 'For the data from Vasselli et al. [I0] two groups of rats (EO-HF and WL-HF) are compared; each group has 49 observations.

2The data from Redmann \& Argyropoulos [14] consists of 16 mice with genotype '+/+' and 21 mice with genotype '-/-'.

\# For Data from Vasselli et al. [10] $\tau$ is set to II0; for data from Redmann \& Argyropoulos [14] $\tau$ is set to 100.

*Only one group has observations above the threshold $\tau$. 
use permutation testing for multiple groups to replace that for two groups.

We have shown that the new methods are effective by simulation studies when the sample size $(\mathrm{N})$ of each group is 50,100 , or 200 . We expect that these methods will be also be relatively more powerful than existing competitors for much larger sample sizes, such as $\mathrm{N}=500$ or even $\mathrm{N}=$ 5000. There are some mouse data sets (like those of the National Institute of Aging's Intervention Testing Program) where $\mathrm{N}>500$, and worm and fly data sets in which $\mathrm{N}$ may sometimes even exceed 5000 . We expect that the new methods are equally applicable to the analysis of such data.

Finally, we note that the tests proposed here are described for the context of testing for differences in lifespan. However, there is nothing intrinsic to them that limits their use to survival data. They could be applied to any situation in which one wanted to test for group differences in the tails of distributions.

\section{Competing interests}

The authors declare they have no competing interests.

\section{Authors' contributions}

DBA participated in all parts of the work of the study (including the study design, methodology development, simulations, data acquisition, and manuscript drafting). He wrote major sections of the original manuscript. He revised final version of the manuscript. DTR provided consulting on the statistical issues in the study and manuscript editing. SZ provided assistance in programming for simulation studies. WW provided consulting on simulation and prepared the figures. GG did all simulation studies and real data analyses and drafted the sections of Results, Illustration with real data, and Discussion of the manuscript and participated in revision of the manuscript.

\section{Acknowledgements}

We thank Richard Miller, David Harrison, and Simon Klebanov for thought provoking dialogue that inspired this paper and George Argyropoulos for graciously providing data. This research was supported in part by $\mathrm{NIH}$ grants P30DK056336, ROIDK067487, and P0IAGII9I5 and by grant GM073766 from the National Institute of General Medical Sciences.

\section{References}

I. Miller RA, Harrison DE, Astle CM, Floyd RA, Flurkey K, Hensley KL, Javors MA, Leeuwenburgh C, Nelson JF, Ongini E, Nadon NL, Warner HR, Strong R: An aging Interventions Testing Program: study design and interim report. Aging Cell 2007, 6(4):565-575.

2. Wanagat J, Allison DB, Weindruch R: Caloric intake and aging: mechanisms in rodents and a study in nonhuman primates. Toxicol Sci 1999, 52(2 Suppl):35-40.

3. Ingram DK, Zhu M, Mamczarz J, Zou S, Lane MA, Roth GS, deCabo $\mathrm{R}$ : Calorie restriction mimetics: an emerging research field. Aging Cell 2006, 5(2):97-108.
4. Weindruch R, Walford RL: The retardation of aging and disease by dietary restriction. In The retardation of aging and disease by dietary restriction C.C. Thomas Publisher, Springfield, IL; 1988.

5. Wang C, Li Q, Redden DT, Weindruch R, Allison DB: Statistical methods for testing effects on "maximum lifespan". Mech Ageing Dev 2004, I 25(9):629-32. Erratum in: Mech Ageing Dev 2006, I27(7):652

6. Redden DT, Fernandez JR, Allison DB: A simple significance test for quantile regression. Stat Med 2004, 23( 16):2587-97.

7. Mehrotra DV, Chan IS, Berger RL: A cautionary note on exact unconditional inference for a difference between two independent binomial proportions. Biometrics 2003, 59:44I-450.

8. Little RC, Folks JL: On the comparison of two methods of combining independent tests. Journal of the American Statistical Association 1972, 67(337):223.

9. Dominici F, Zeger SL: Smooth quantile ratio estimation with regression: estimating medical expenditures for smokingattributable diseases. Biostatistics 2005, 6:505-5I9.

10. Vasselli JR, Weindruch R, Heymsfield SB, Pi-Sunyer FX, Boozer CN, Yi N, Wang C, Pietrobelli A, Allison DB: Intentional weight loss reduces mortality rate in a rodent model of dietary obesity. Obes Res 2005, I3(4):693-702.

II. Wilcoxon F: Individual comparisons by ranking methods. Biometrics 1945, I:80-83.

12. Mann HB, Whitney DR: On a test of whether one of two random variables is stochastically larger than the other. Annals of Mathematical Statistics 1947, 18:50-60.

13. Good P: Permutation Tests: A Practical Guide to Resampling Methods for Testing Hypotheses. New York: Springer-Verlag; 1994.

14. Redmann SM Jr, Argyropoulos G: AgRP-deficiency could lead to increased lifespan. Biochem Biophys Res Commun 2006, 35 I (4):860-4.

\section{Pre-publication history}

The pre-publication history for this paper can be accessed here:

http://www.biomedcentral.com/1471-2288/8/49/prepub
Publish with Biomed Central and every scientist can read your work free of charge

"BioMed Central will be the most significant development for disseminating the results of biomedical research in our lifetime. " Sir Paul Nurse, Cancer Research UK

Your research papers will be:

- available free of charge to the entire biomedical community

- peer reviewed and published immediately upon acceptance

- cited in PubMed and archived on PubMed Central

- yours - you keep the copyright

Submit your manuscript here:

http://www.biomedcentral.com/info/publishing_adv.asp
BioMedcentral 